\title{
Special issue on multimedia in ecology
}

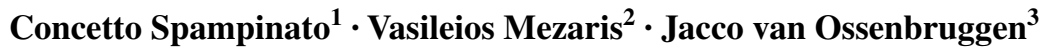

(C) Springer-Verlag Berlin Heidelberg 2016

With the recent progress in digital cameras and sensors, as well as in network bandwidth and information storage capacities, the production of multimedia data has become an easy task. This has resulted in a huge amount of multimedia available on the Web, in broadcast data streams, or in personal and professional databases. This explosion of multimedia data has created the urgent need for efficient organisation, browsing, retrieval, and visualisation tools. It has also generated new possibilities for exploiting multimedia data in diverse and specialised applications that can significantly gain from the analysis and understanding of such data, such as ecology. Indeed, recently we have witnessed the proliferation of ecology-related multimedia content, e.g., many Terabytes of data (videos, images, and audio recordings) for monitoring forest animals and marine organisms, plants, etc., have been recorded. The automated analysis of such multimedia data poses new challenges, and the results of such analyses are of great interest to investigators, such as biologists, in their strive towards monitoring and analysing the natural environment, promoting its preservation, and understanding the behavior and interactions

Concetto Spampinato

cspampin@dieei.unict.it

Vasileios Mezaris

bmezaris@iti.gr

Jacco van Ossenbruggen

Jacco.van.Ossenbruggen@cwi.nl

1 Department of Computer Engineering, University of Catania, Catania, Italy

2 Centre for Research and Technology Hellas, Thessaloniki, Greece

3 Centre of Mathematics and Informatics, Amsterdam, The Netherlands of the living organisms (insects, animals, etc.) that are part of it. This special issue aims at supporting this goal by reporting a shortlist of the most recent methods for processing, annotating, retrieving, and visualising ecological data and its analysis:

- Tian et al. in "Motion analytics of zebrafish using fine motor kinematics and multi-view trajectory" present an automatic method for zebrafish motion analytics with the objective to distinguish behavior between wild-type (normal) and transgenic zebrafish. In particular, the proposed framework first extracts the quantitative measurements of motor movement using a high-frame rate camera (up to 1000 frames per second). These motion cues are then used by a linear SVM classifier to identify abnormal zebrafish trajectories with an accuracy, expressed as average recognition rate, of about $80 \%$.

- Palazzo et al. in "A diversity-based search approach to support annotation of a large fish image data set" propose an image retrieval approach able to reduce significantly near-duplicates when querying large data sets. More specifically, the authors present a method which favors the retrieval of as many different views of the query image as possible. It relies on a diversity-based clustering technique using a random-forest framework combined to a label propagation approach able to efficiently retrieve images at large scale. The method, tested on a very large data set of fish images, reached the promising performance in image retrieval, ensuring diversification of the annotated items while preserving precision.

- Beauxis-Aussalet et al. in "Uncertainty-aware estimation of population abundance using machine learning" propose a method able to improve fish image classification accuracy using limited ground-truth. Furthermore, 
a visualisation tool for understanding and evaluating classification uncertainty is also introduced and evaluated to support end-users in understanding/interpreting misclassifications. Despite the method was tested on a marine scenario to support fish population abundance investigations, it can be applied to a large variety of systems using machine learning technologies.

- Joly et al. in "A look inside the Pl@ntNet experience: the good, the bias and the hope" provide a thorough analysis and a critical evaluation of 1-year experience from the release (as a part of the participatory sensing platformPl@ntNet) of a mobile application for imagebased plants identification. The authors first present the requirements for sustainable and effective ecological surveillance tools, demonstrating the attractiveness and the collaborative capacity that they may have in collecting ecological data. Afterwards, the authors discuss the limitations of their method in generating accurate distribution maps of image plants at a very large scale, focusing on two key aspects: the bias and the incompleteness of user-contributed data.

- Torres et al. in "Habitat image annotation with lowlevel features, medium-level knowledge, and location information" face the challenging problem of habitats classification (usually performed through expensive and error-prone manual surveys), which is a important step to understand the natural world. The authors tackle the problem as a fine-grained visual categorisation one and propose a random-forest-based method that takes into consideration visual and geographical closeness for classification. For classifier training, beside lowlevel visual cues, medium-level contextual information is employed. Such information is extracted through a human-in-the-loop methodology which asks non-expert a set of questions about the image appearances. The performance analysis showed that the proposed method was able to classify with a reasonable degree of confidence four of the main habitat classes: Woodland and Scrub, Grassland and Marsh, Heathland, and Miscellaneous. Furthermore, the authors also present a georeferenced habitat image database containing over 1000 high-resolution ground images manually annotated by habitat classification experts.

Our thanks go, first, to the authors for their contribution to this special issue and, then, to the reviewers for the effort and time spent to provide thorough reviews and valuable advice to the submitted manuscripts. We would also like to extend thanks to the Editor in Chief, Professor Thomas Plagemann, and the whole editorial staff of Multimedia
Systems Journal for greatly supporting us during the whole special issue process and for understanding and recognising the importance that this special issue may have on future research on multimedia and ecology research area, whose development is, in our opinion, of crucial importance to comprehend the world which we live in and how we can exploit sustainably its resources.

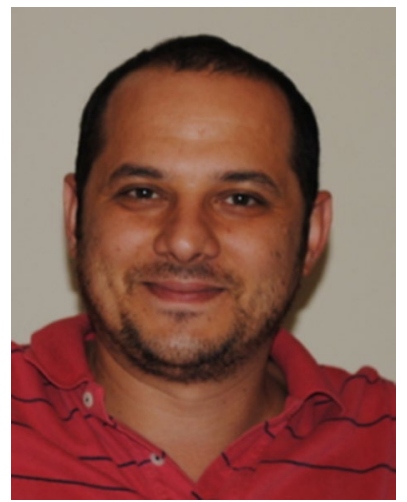

Concetto Spampinato received the Laurea (grade $110 / 110$ cum laude) degree in computer engineering and the $\mathrm{Ph} . \mathrm{D}$. degree from the University of Catania, Catania, Italy, in 2004 and 2008, respectively, where he is currently an assistant professor. His research interests include mainly computer vision and pattern recognition with a particular focus on ecological data analysis. $\mathrm{He}$ is one of the pioneers, being involved in the EU project Fish4Knowledge, of underwater computer vision, i.e., the development of computer vision solutions for monitoring sea and ocean biodiversity. He has coauthored over than 100 publications in international refereed journals and conference proceedings. As further research activities, he has organised and chaired dedicated workshops on multimedia in ecology (MAED 2012, 2013, 2014) several special sessions at mainstream conferences and guest-edited six special issues of international journals with impact factor. He is a member of the Editorial Board of Ecological Informatics journal.

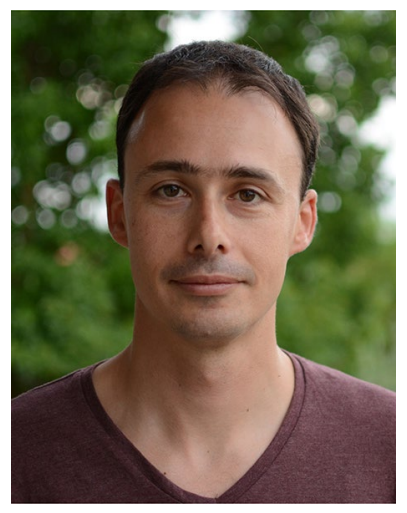

Vasileios Mezaris received the B.Sc. and Ph.D. in Electrical and Computer Engineering from the Aristotle University of Thessaloniki, in 2001 and 2005, respectively. He is a Senior Researcher (Researcher B) at the Information Technologies Institute (ITI) of the Centre for Research of Technology Hellas (CERTH). His research interests include image and video analysis, event detection in multimedia, machine learning for multimedia analysis, content-based and semantic image and video retrieval, and applications of image and video analysis in specific domains (TV broadcasting and News, medical images, ecological data, educational, and cultural applications). He has coauthored more than 30 papers in refereed journals, 10 book chapters, 130 papers in international conferences, and 3 patents. He served as an Associate Editor for the IEEE Transactions on Multimedia (2012-2015), serves as an Associate Editor for the IEEE Signal Processing Letters, and is a Senior Member of the IEEE. 


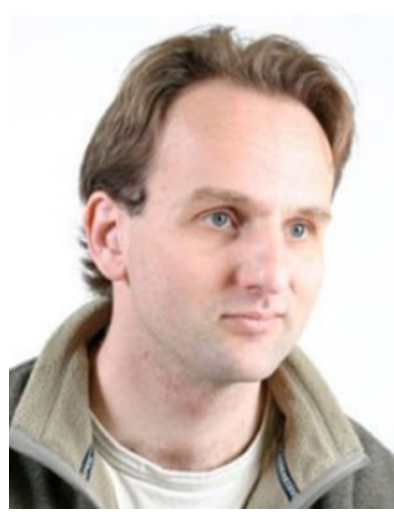

Jacco van Ossenbruggen is a senior researcher with the Interactive Information Access group at the Centrum voor Wiskunde en Informatica (CWI) in Amsterdam, and affiliated as an assistant professor with the Web and media research group at VU University in Amsterdam. He obtained a $\mathrm{PhD}$ in computer science from VU University Amsterdam in 2001. His research interests include semantic Web interfaces for cultural heritage and other linked open data (working with Riste Gligorov, Alia Amin and Michiel Hildebrand on interfaces such as facet) and Web-based data integration (working with AnnaTordai on alignment of SKOS vocabularies). Jacco is currently active in the Agora and PrestoPrime projects and the Europeana Connect Best Practice Network. 